Trauma Berufskrankh 2014 - 16[Suppl 3]:287-291 DOI 10.1007/s10039-014-2094-2

Online publiziert: 18. Juli 2014

c) Springer-Verlag Berlin Heidelberg 2014

O. Bleiziffer · M. Sauerbier

Abteilung für Plastische, Hand- und Rekonstruktive Chirurgie,

Berufsgenossenschaftliche Unfallklinik Frankfurt am Main

\title{
Infektionen der Hand und des Unterarms
}

\section{Beuge- und Strecksehnen}

schließen sich eine keimgerechte Antibiotikatherapie sowie Ruhigstellung und frühfunktionelle Beübung an. Trotz adäquater und zeitgerechter Therapie lassen sich dauerhafte Folgeschäden nicht immer vermeiden. Komplikationen durch Infektionen von Hand- und Unterarm schließen Gelenkinfekte (Empyeme) der Fingergelenke bzw. des Handgelenks ein, ebenso generalisierte Infektzeichen bis hin zur Sepsis. Wundheilungsstörungen gehen v. a. bei Patienten mit entsprechender Prädisposition und Risikofaktoren nicht selten mit Nekrosen von Haut und Weichteilen einher. Falls es zur Exposition vitaler Strukturen wie Sehnen, Nerven und Blutgefäßen kommt, wird im Regelfall eine Defektdeckung mit Hilfe plastisch-rekonstruktiver Maßnahmen, insbesondere Lappenplastiken, erforderlich.

Im Folgenden werden Ätiologie und klinischer Verlauf, Diagnostik und Therapie von Infektionen an Hand und Unterarm ebenso detailliert beleuchtet wie Outcome, Komplikationen und deren Management.

\section{Ätiologie und klinischer Verlauf} schweren können, wie Immunsuppression, z. B. durch Kortisondauertherapie, Gicht, rheumatische Grunderkrankungen sowie Diabetes mellitus.

Eine adäquate operative Behandlung beinhaltet die chirurgische Entlastung mittels Inzision, Weichgewebeexzision bzw. radikalem Débridement, Spülung und Drainage. Im weiteren Verlauf
Der Auslöser einer Handinfektion lässt sich nicht in jedem Einzelfall mit letzter Sicherheit eruieren. Zumeist liegt allerdings eine Verletzung der Hand durch Schnitte, Stiche, Fremdkörperinokulationen (z. B. Holzspreißel, Dorn, Metallsplitter), Bissverletzungen, v. a. durch Hunde, Katzen, aber auch Menschen, zugrunde.
In derartigen Fällen sind je nach Lokalisation der Verletzung eine genaue Funktionsprüfung von Beuge- und Strecksehnen und die Evaluation der Sensibilität mittels 2-Punkt-Diskrimination besonders wichtig, damit Begleitverletzungen nicht übersehen werden.

Die Hand zeigt einige Charakteristika im anatomischen Aufbau, die für das Verständnis der Entstehung von Handinfektionen, insbesondere Handphlegmonen, unerlässlich sind und die typischen Ausbreitungswege von der Oberfläche in tiefere Gewebeschichten und von peripher nach zentral bedingen. Durch eine Hautläsion werden Keime inokuliert und gelangen durch vertikal orientierte Bindegewebssepten auf der Beugeseite rasch von der Oberfläche in die Tiefe. Nach Durchdringen der Palmarfaszie können sie auch in die Sehnenscheiden penetrieren und sich über diese, die dann sozusagen als Infektstraßen dienen, von peripher nach zentral ausbreiten. Ein schneller Übertritt der Keime auf die tiefen Hohlhandkompartimente ist über eine subfasziale Ausbreitung möglich. Von dort können die Erreger in den Unterarm fortgeleitet werden, da die tiefen Kompartimente von Hand und Unterarm miteinander in Verbindung stehen ( $\bullet$ Abb. 1).

Klinisch zeigen sich die typischen Zeichen einer Entzündung, die zunächst lokal begrenzt sind:

- Schwellung,

- Rötung und

- Druckschmerzhaftigkeit. 


\section{Hand- und Unterarminfektionen}

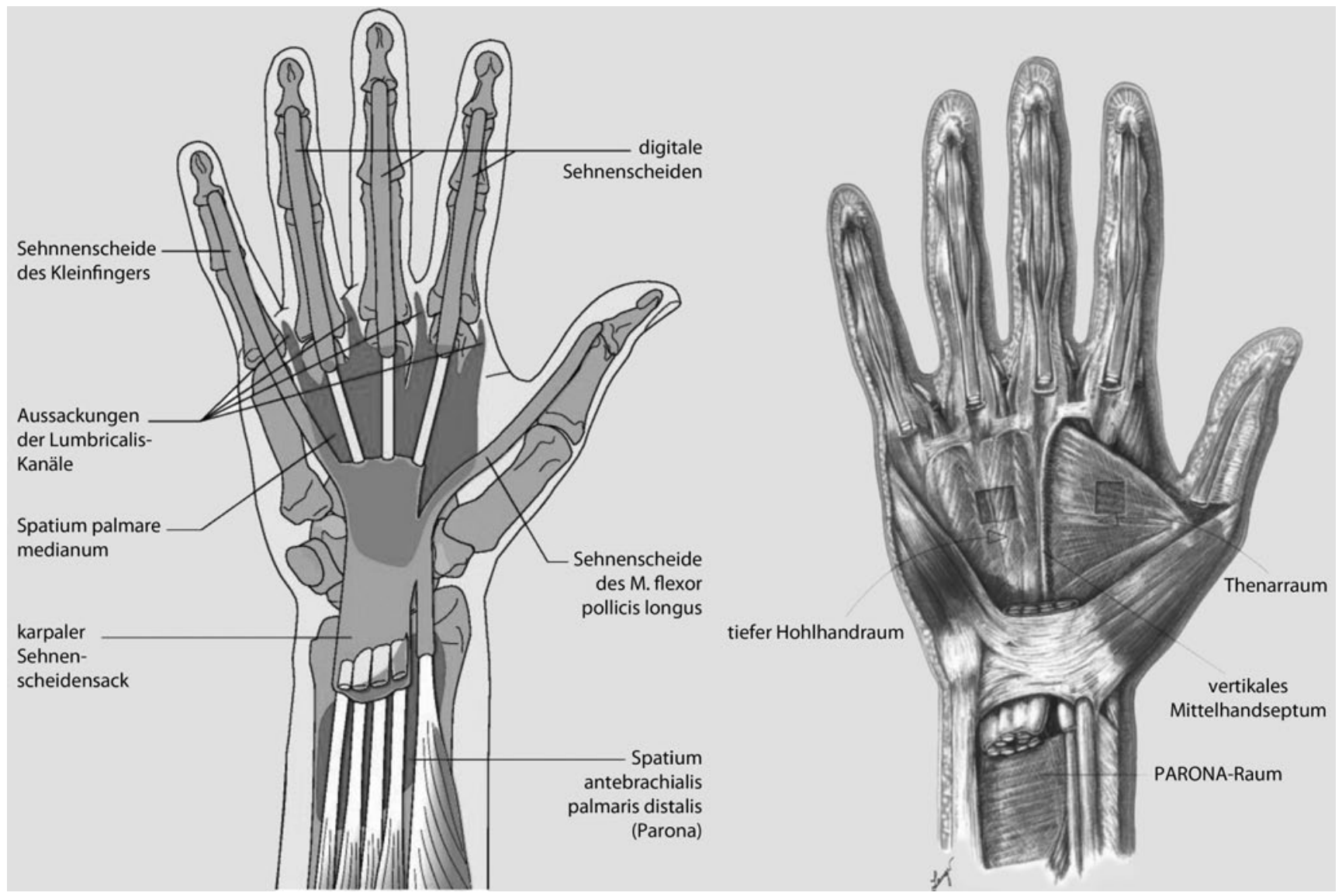

Abb. $1 \Delta$ Topografische Verhältnisse der Sehnenscheiden, Faszien und weichgeweblichen Schichten an beugeseitigem Unterarm und der Hohlhand, aufgrund der Kommunikation dieser Räume schnelle Ausbreitung von Infektionen möglich. (Aus [4], mit freundl. Genehmigung von Thieme)

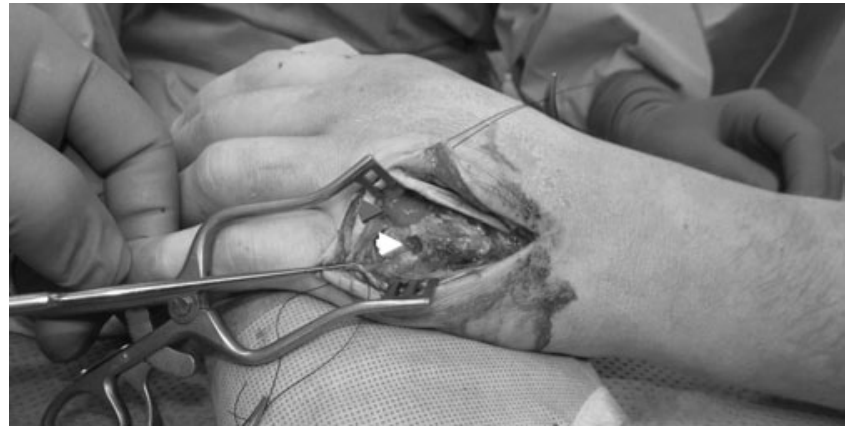

Abb. $2 \Delta$ Charakteristisches Bild einer eitrigen Handphlegmone nach Hundebissverletzung im Bereich der ellenseitigen Mittelhand mit Affektion des 5. Mittelhandknochens (weißer Pfeil) und sich entleerendem eitrigem Sekret (grauer Pfeil), Strecksehnen mit einem Haken beiseite gehalten: bestehende Indiaktion zum radikalen Débridement einschließlich Synovialektomie der Strecksehnen

Eine Fluktuation kann eine subkutane Eiteransammlung nahelegen. Bei weiter fortgeschrittener Infektion finden sich

- Lymphangitis,

- Lymphadenitis,

- Fieber und

- ein ausgeprägtes Krankheitsgefühl.
Bei Beteiligung der Beugesehnenscheiden wird der Finger des betroffenen Strahls in Schonhaltung flektiert gehalten. Dessen passive Streckung induziert beim Patienten einen heftigen Schmerz und muss als für eine Beugesehnenscheidenphlegmone hochverdächtig gelten. Diese bedarf einer operativen Revision. Da die Beugesehnenscheiden des Daumens und des Kleinfingers kommunizieren, findet sich gelegentlich eine Beugesehnscheidenphlegmone mit Beteiligung des Daumen- und Kleinfingerstrahls, die als V-Phlegmone bezeichnet wird [4].

Auf das Vorhandensein eines axialen Kompressionsschmerzes von Fingergelenken oder des Handgelenks als dringendem Hinweis auf das Vorliegen eines Gelenkempyems ist besonders zu achten. Im Zweifelsfall ist intraoperativ die Gelenkrevision mit Inspektion und ggf. Drainage eines affektierten Gelenks indiziert.

Bei länger dauerndem Verlauf und konservativer Therapie, insbesondere bei frustraner konservativer Therapie unter Antibiotikagabe, ist die potenzielle knöcherne Beteiligung in Betracht zu ziehen. Hier sollte auf jeden Fall die röntgenologische Abklärung mittels konventioneller Röntgenaufnahmen, falls erforderlich auch MRT (Magnetresonanztomografie) 
und CT (Computertomografie) erfolgen, um ggf. eine Osteomyelitis zu detektieren, die durch charakteristische Osteolysen als Nachweis einer fortgeschrittenen Knochendestruktion imponiert.

Neben der überwiegenden Genese durch Schnitt- und Stichverletzungen können auch Bissverletzungen durch Tiere, v. a. Hunde und Katzen, aber auch Menschen, zu einer Infektion an Hand und Unterarm führen. Diese stellen einen Sonderfall und eine besondere Herausforderung für den klinisch tätigen Arzt dar, da einige Besonderheiten zu beachten sind. Zum einen kann durch das etwas abweichende Keimspektrum die antibiotische Behandlung aufgrund auftretender Resistenzen problematisch sein, v. a. bei Plegmonen durch Katzenbisse. Zum anderen kann es zu charakteristischen Begleitverletzungen von Sehnen, Nerven oder Knochen kommen, die bei einer klinisch apparenten und gleichzeitig auftretenden Infektion übersehen werden können. Beispielsweise besteht bei Hautläsionen im Bereich der Metakarpalköpfchen und der MCP-Gelenke (MCP: Metacarpophalangealgelenk) immer der Verdacht auf einen gegen die Zahnreihe ausgeübten Faustschlag als Ursache. Durch einen solchen kann es sowohl zu Frakturen als auch zu einer knöchernen Beteiligung durch einen Infekt im Sinne einer Osteomyelitis kommen. Ein charakteristisches Bild einer eitrigen Phlegmone nach Hundebissverletzung an der ulnarseitigen Mittelhand mit knöcherner Beteiligung des Kopfs des Os metacarpale 5 zeigt $\bullet$ Abb. 2.

\section{Keime und antibiotische Therapie}

Das Keimspektrum bei Weichteilinfektionen an Hand und Unterarm ist breit gefächert. Bei sog. konventionellen Phlegmonen, die durch Verletzungen im häuslichen Umfeld oder bei der Arbeit entstehen, ist allerdings in der überwiegenden Mehrzahl der Fälle (50-80\%) Staphylococcus aureus als Verursacher mikrobiologisch im Wundabstrich oder in der Gewebeprobe nachweisbar, nicht selten finden sich dabei auch MRSA (methicillinresistenter Staphylococcus aureus, [3]). Wann immer möglich sollte nicht nur ein

Trauma Berufskrankh 2014 · 16[Suppl 3]:287-291 DOI 10.1007/s10039-014-2094-2

(c) Springer-Verlag Berlin Heidelberg 2014

\section{O. Bleiziffer $\cdot$ M. Sauerbier \\ Infektionen der Hand und des Unterarms. Beuge- und Strecksehnen}

\section{Zusammenfassung}

Hintergrund. Weichteilinfektionen an Hand und Unterarm gehören zu den häufigsten handchirurgischen Krankheitsbildern. Sie treten oft nach Bagatellverletzungen, seltener aufgrund hämatogener Streuung auf, können protrahiert verlaufen und in einer bleibenden Funktionseinschränkung der betroffenen Hand resultieren. Deshalb ist eine unverzügliche adäquate Therapie, die nicht selten eine oder mehrere operative Interventionen erfordert, unerlässlich.

Diagnostik. Bei jedem Verdacht auf eine Infektion an der Hand ist eine genaue symptomorientierte Anamnese mit klinischer Untersuchung und weiterführenden Laborund ggf. auch Röntgenuntersuchungen indiziert. Begleitverletzungen dürfen nicht übersehen werden, und Begleiterkrankungen, die den Verlauf der Infektion erschweren können, sollten abgeklärt werden.

Therapie. Unbedingt ist die rasche Einleitung einer empirischen Antibiotikatherapie mit gut gewebegängigen, ein breites Keimspektrum abdeckenden und v. a. hinsichtlich Staphylococcus aureus gut wirksamen Agenzien zu empfehlen. Diese sollte nach Vorliegen eines Antibiogramms rasch auf eine keimgerechte Antibiotikatherapie umgestellt werden. Wesentliche Bestandteile der operativen Behandlung sind die chirurgische Entlastung mittels Inzision, Weichgewebeexzision bzw. radikalem Débridement. Postoperativ schließen sich eine Ruhigstellung in einer Gipsschiene, eine möglichst frühzeitig beginnende funktionelle Beübung sowie die Wundspülung und eine möglichst keimgerechte Antibiotikabehandlung an. Komplikationen sind auch bei adäquater Therapie nicht immer vermeidbar.

\section{Schlüsselwörter}

Weichgewebeinfektionen · Hand · Unterarm · Antibiotika - Operative Therapie

\section{Infections of the hand and forearm. Flexor and extensor tendons}

\section{Abstract}

Background. Soft tissue infections of the hand and forearm belong to most frequently occurring syndromes in hand surgery. They often arise following minor injuries and less commonly due to hematogenic spreading, they can have a protracted course and result in permanent functional limitations of the affected hand. Therefore, an immediate and adequate treatment which not uncommonly requires one or more operative interventions, is imperative.

Diagnostics. For every suspected infection of the hand a detailed symptom-oriented medical history with clinical examinations and extensive laboratory and if necessary $X$ ray investigations are indicated. Accompanying injuries must not be overlooked and comorbidities which could complicate the course of the infection must be clarified.

Therapy. The rapid induction of an empirical broad spectrum antibiotic therapy with good tissue permeating agents and in particular good effectiveness with respect to Staphylococcus aureus is absolutely recommended. This should be rapidly switched to a pathogen-specific antibiotic therapy when an antibiogram has been carried out. Essential components of the operative treatment are surgical relief by incision and soft tissue excision or radical débridement. This is followed by postoperative immobilization in a plaster cast, functional physiotherapy as early as possible, wound irrigation and if possible a pathogen-specific antibiotic therapy. Complications are not always avoidable even after adequate therapy.

\section{Keywords}

Soft tissue infections · Hand · Forearm · Antibacterial agents Operative therapy
Wundabstrich, sondern eine Gewebeprobe mikrobiologisch aufgearbeitet werden, da hierdurch gerade bei antibiotisch anbehandelten Patienten die Wahrscheinlichkeit eines positiven Keimnachweises erhöht wird.
Aufgrund der erwähnten statistischen Wahrscheinlichkeit empfiehlt es sich, unbedingt eine empirische Antibiotikatherapie mit Agenzien einzuleiten, die gut gewebegängig sind, ein breites Keimspektrum abdecken und v. a. hinsichtlich des 
Tab. 1 Antibiotika der Wahl bei Weichteilinfektionen an Hand und Unterarm. (Nach [1, 2, 5, 6])

\begin{tabular}{|c|c|c|c|c|c|}
\hline \multicolumn{3}{|c|}{ Antibiotikum } & Wirkspektrum & Indikation & Vor- und Nachteile \\
\hline \multirow[t]{2}{*}{$\begin{array}{l}\text { Zephalo- } \\
\text { sporine }\end{array}$} & Gruppe 1 & $\begin{array}{l}\text { Cefazolin } \\
\text { (Elzogram }^{\oplus} \text {; i.v.) } \\
\text { Cefachlor } \\
\text { (Panoral } \text {; oral) } \\
\text { Cefalexin } \\
\text { (Cephalexin }{ }^{\oplus} \text {; oral) }\end{array}$ & $\begin{array}{l}\text { Grampositive Bakterien: } \\
\text { u. a. Staphylokokken } \\
\text { Gramnegative Bakterien: } \\
\text { u. a. Enterobakterien (E.coli/Klebsiellen) }\end{array}$ & & \\
\hline & Gruppe 2 & $\begin{array}{l}\text { Cefuroxim } \\
\text { (Zinacef }{ }^{\oplus} ; v_{.} \text {) } \\
\text { Cefuroxim-Axetil } \\
\text { (Zinnat }{ }^{\oplus} ; \text { oral) }\end{array}$ & $\begin{array}{l}\text { Grampositive Bakterien: } \\
\text { u. a. Staphylokokken, Streptokokken der } \\
\text { Gruppen A und B } \\
\text { Gramnegative Bakterien: u. a. Entero- } \\
\text { bakterien (E.coli/Klebsiellen/Proteus) }\end{array}$ & & \\
\hline Penizilline & \multicolumn{2}{|c|}{$\beta$-Laktam-Antibiotika } & $\begin{array}{l}\text { Große therapeutische Breite gegenüber } \\
\text { grampositiven und -negativen Kokken } \\
\text { und Stäbchen }\end{array}$ & $\begin{array}{l}\text { Unter ande- } \\
\text { rem Wund- } \\
\text { infektionen }\end{array}$ & $\begin{array}{l}\text { Schlecht gewebe-/knochengängig } \\
\text { Hauptkeim Staphylococcus aureus bis zu } \\
\sim 80 \% \text { penizillinresistent ( } \beta \text {-Laktamase) } \\
\rightarrow \text { Anwendung von Antibiotika mit } \\
\text { geringerer Wirkintensität: } \\
\text { Flucloxacillin }\left(\text { stapylex }^{\oplus} \text { ) }\right. \\
\text { Dicloxacillin (InfectoStaph }{ }^{\oplus} \text { ) } \\
\text { Amoxycillin/Clavulansäure (Augmentan }{ }^{\oplus} \text { ) } \\
\text { Ampicillin/Sulbactam (Unacid }{ }^{\oplus} \text { ) }\end{array}$ \\
\hline \multicolumn{6}{|c|}{ Therapie der 2. Wahl } \\
\hline \multirow{4}{*}{$\begin{array}{l}\text { Fluor- } \\
\text { chinolone }\end{array}$} & Gruppe 1 & Norfloxacin & & \multirow{4}{*}{$\begin{array}{l}\text { Unter ande- } \\
\text { rem Wund- } \\
\text { infektionen }\end{array}$} & \multirow[t]{4}{*}{ Gut gewebe-/knochengängig } \\
\hline & Gruppe 2 & $\begin{array}{l}\text { Ciprofloxacin } \\
\text { (Cibrobay }^{\circledast} \text { ) } \\
{\text { Ofloxacin }\left(\text { Tarivid }^{\circledR} \text { ) }\right.}\end{array}$ & $\begin{array}{l}\text { Gramnegative Bakterien (inklusive Pseu- } \\
\text { domonas, Enterobakterien) schwächer } \\
\text { gegen Staphylokokken, Enterokokken }\end{array}$ & & \\
\hline & Gruppe 3 & $\begin{array}{l}\text { Levofloxacin } \\
\text { (Tavanic }^{\oplus} \text { ) }\end{array}$ & $\begin{array}{l}\text { Verbesserte Aktivität gegenüber } \\
\text { grampositiven Erregern (Staphylo-, } \\
\text { Streptokokken, Enterokokken) }\end{array}$ & & \\
\hline & Gruppe 4 & $\begin{array}{l}\text { Moxifloxacin } \\
\left(\text { Avalox }{ }^{\oplus}\right)\end{array}$ & $\begin{array}{l}\text { Wie Gruppe 3, zusätzlich gegen } \\
\text { Anaerobier wirksam }\end{array}$ & & \\
\hline
\end{tabular}

häufigsten Verursachers, Staphylococcus aureus, gut wirksam sind. Unter diesem Gesichtspunkt sind Zephalosporine der Gruppe 1 und 2 Mittel der 1. Wahl. Alternativ können, v. a. bei Allergien gegen Zephalosporine, die gut gewebe- und knochengängigen Fluorchinolone zum Einsatz kommen. Allerdings sollte möglichst schnell nach Vorliegen eines Antibiogramms auf eine keimgerechte Antibiotikatherapie umgestellt werden. Eine Übersicht über die gängigsten Antibiotika und deren Keimspektrum findet sich in $\bullet$ Tab. 1.

\section{Operative Therapiestrategien bei Infektionen an Hand und Unterarm}

Nicht selten ist die chirurgische Intervention zur Beherrschung einer phlegmonösen Infektion an den Weichteilen von Unterarm und Hand unabdingbar, und bei der Indikationsstellung muss die Nichtoperationspflichtigkeit begrün- det sein. Trotz des breiten Spektrums an Schweregrad, anatomischer Lokalisation und Ausprägung gibt es einige allgemeine Prinzipien der operativen Behandlung, die im Folgenden erwähnt werden sollen.

Die Operation erfolgt in Oberarmblutsperre. Eine Blutleere sollte nicht eingesetzt werden, um einer Keimverschleppung vorzubeugen. Ebenso dringlich ist die Lupenbrillenvergrößerung, um eine Verletzung der kleinen Gefäße und Nerven, v. a. in der Hohlhand, zu vermeiden bzw. diese Strukturen adäquat darstellen zu können. Vor allem bei protrahierten Verläufen finden sich häufig nekrotisch eingeschmolzene Gewebeareale, welche konsequent und radikal debridiert werden müssen, um eine zügige Ausheilung zu ermöglichen. Dazu gehören auch eitrige Synovialitiden der Sehnenscheiden. In schweren Fällen können die Sehnen selbst lytisch verändert sein und sind dann ggf. nicht vollständig zu erhalten. Ebenso muss danach getrachtet werden, adäquate Gewebeproben und Wundab- striche zu nehmen, um die Chance auf einen Keimnachweis in der mikrobiologischen Untersuchung zu erhöhen.

Postoperativ schließen sich eine $\mathrm{Ru}$ higstellung in einer Gipsschiene in anatomiegerechter Position, eine möglichst frühzeitig zu beginnende funktionelle Beübung sowie die Wundspülung mit einer Drainage, ggf. auch als Spül-Saug-Drainage, an. Dagegen ist die Anwendung von Laschen heute obsolet, da es sich hierbei um offene Ablaufsysteme handelt, wodurch das Kontaminationsrisiko erhöht werden kann. In den ersten Tagen nach Operation einer Phlegmone an Hand und Unterarm sind tägliche Verbandswechsel und Befundkontrollen obligat, um ggf. bei einer Befundverschlechterung zeitnah einen operativen „second look“ folgen zu lassen.

Auch und gerade bei konservativem Vorgehen sollte unbedingt eine Kontinuität der Befundkontrolle und Weiterbehandlung gegeben sein, auch z. B. an Wochenenden und Feiertagen, um, falls er- 
forderlich, rechtzeitig operativ intervenieren zu können.

\section{Komplikationen}

Trotz aller erwähnten Maßnahmen können auch bei optimaler Therapie nicht immer eine vollständige Restitutio ad integrum und Wiederherstellung der Funktion gewährleistet werden. Im schlimmsten Fall droht nach Arbeitsunfällen auch eine Minderung der Erwerbsfätigkeit (MdE) in rentenberechtigendem Ausmaß.

\section{Fazit für die Praxis}

- Infektionen der Weichteile an Hand und Unterarm können in ihrer Behandlung eine große Herausforderung darstellen.

- Infektionen der Weichteile an Hand und Unterarm müssen von entsprechend erfahrenen Spezialisten behandelt werden.

\section{Korrespondenzadresse}

\section{Dr. O. Bleiziffer}

Abteilung für Plastische, Hand-

und Rekonstruktive Chirurgie, Berufsgenossenschaftliche Unfallklinik Frankfurt am Main, Friedberger Landstraße 430, 60389 Frankfurt am Main oliveranton.bleiziffer@bgu-frankfurt.de

\section{Einhaltung ethischer Richtlinien}

Interessenkonflikt. O. Bleiziffer und M. Sauerbier geben an, dass kein Interessenkonflikt besteht.

Dieser Beitrag beinhaltet keine Studien an Menschen oder Tieren.

The supplement containing this article is not sponsored by industry.

\section{Literatur}

1. Deutsche Dermatologische Gesellschaft, Berufsverband Deutscher Dermatologen, Deutsche Gesellschaft für Infektiologie (DGI), Deutsche Gesellschaft für Hygiene und Mikrobiologie (DGHM), Deutsche Gesellschaft für Pädiatrische Infektiologie (DGPI), Paul Ehrlich Gesellschaft für Chemotherapie (PEG) (2011) Staphylococcus aureus bedingte Infektionen der Haut und Schleimhäute, Diagnostik und Therapie. AWMF-Leitlinienregisternummer 013-038. AWMF, Düsseldorf. http://www. awmf.org/uploads/tx_szleitlinien/013-038I_S2k_ Staphyococcus_aureus_2011-09.pdf. Zugegriffen: 14.05.2014

2. Hardman JG, Limbird LE, Gilman AG (Hrsg) (2001) Goodman \& Gilman's the pharmacological basis of therapeutics, 10. Aufl. McGraw-Hill, New York

3. Kowalski TJ, Thompson LA, Gundrum JD (2014) Antimicrobial management of septic arthritis of the hand and wrist. Infection 42:379-384

4. Pillukat T, Schädel-Höpfner M, Windolf J, Schoonhoven J van (2013) Infektionen der Beugesehnenscheiden. Handchir Scan 2:217-231

5. Stevens DL, Bisno AL, Chambers HF et al; Infectious Diseases Society of America (2005) Practice guidelines for the diagnosis and management of skin and soft-tissue infections. Clin Infect Dis 41:13731406

6. Wongworawat MD, Schnall SB (2002) Hand infections. Curr Treat Options Infect Dis 4:295-301 